\title{
Circular RNA SMARCA5 correlates with favorable clinical tumor features and prognosis, and increases chemotherapy sensitivity in intrahepatic cholangiocarcinoma
}

\section{Lu Qi}

Department of Hepatobiliary and Pancreatic Surgery, Huangshi Central Hospital (Affiliated Hospital of Hubei Polytechnic University)

Fang Tao ( $\square$ taoke915881643@163.com )

Huangshi Central Hospital (Affiliated Hospital of Hubei Polytechnic University)

\section{Research article}

Keywords: Circular RNA SMARCA5, Prognosis, Cell proliferation, Chemotherapy sensitivity, Intrahepatic cholangiocarcinoma

Posted Date: November 13th, 2019

DOl: https://doi.org/10.21203/rs.2.17259/v1

License: (c) (1) This work is licensed under a Creative Commons Attribution 4.0 International License.

Read Full License 


\section{Abstract}

Background This present study aimed to investigate the correlation of circular RNA SMARCA5 (circSMARCA5) with clinicopathological features and overall survival (OS), and the effect of circ-SMARCA5 on cell proliferation and chemotherapy sensitivity to cisplatin/gemcitabine in intrahepatic cholangiocarcinoma (ICC).

Methods Totally 92 primary ICC patients who underwent resection were recruited, and their tumor tissues, adjacent tissues were collected for circ-SMARCA5 detection. The effect of circ-SMARCA5 on cell proliferation and chemotherapy sensitivity was detected after circ-SMARCA5 overexpression plasmids transfection into TFK-1 and HuH-28 ICC cells.

Results Circ-SMARCA5 expression was reduced in ICC tumor tissues compared to adjacent tissues. Tumor circ-SMARCA5 high expression was negatively associated with Eastern Cooperative Oncology Group performance score, T stage, N stage, TNM stage and abnormal CA199 status. Furthermore, OS was increased in patients with tumor circ-SMARCA5 high expression compared with those with low expression, and further multivariate Cox's regression demonstrated that tumor circ-SMARCA5 high expression was an independent predictive factor for longer OS. In TFK-1 and HuH-28 ICC cells, circSMARCA5 upregulation decreased cell proliferation, reduced relative cell viability in cisplatin-treated as well as gemcitabine-treated cells, and also decreased inhibitory concentration by $50 \%$ value (IC50) of cisplatin and gemcitabine.

Conclusions The correlation of circ-SMARCA5 with favorable clinical tumor features, survival profile, and its promoting effect on chemotherapy sensitivity imply its potential as a valuable biomarker in monitoring disease progression and prognosis of ICC.

\section{Background}

Intrahepatic cholangiocarcinoma (ICC), as the second most common primary liver cancer, is a hepatobiliary malignancy located proximally to the second degree bile ducts, and its incidence is increasing in the last twenty years [1,2]. Surgical resection is considered to be the only potentially curative treatment with 5-year survival rates ranging from $30 \%-40 \%$, and remains as the cornerstone of therapy for ICC patients [3]. Besides, other treatments consist of neoadjuvant/adjuvant therapy, intensive cytotoxic therapy, hepatic arterial infusion therapy, embolization therapy and etc., which help improve clinical outcomes in the ICC patients [4-7]. However, there are still a large number of ICC patients suffering from poor long-term survival due to a high risk of recurrence, node metastasis, vascular invasion and increasing chemotherapy resistance $[3,5]$. Therefore, research focusing on the discovery of novel biomarkers is essential, which can be used in the clinical application and improve the long-term therapeutic efficacy.

Circular RNAs (circRNAs) represent a large amount of noncoding RNA transcripts, whose biology functions in cellular differentiation, physiological homeostasis and even tumorigenesis are revealed by 
accumulating publications in the last decade [8]. CircRNA SWI/SNF related, matrix associated, actin dependent regulator of chromatin, subfamily a, member 5 (circ-SMARCA5) is a circRNA derived from exons 15 and 16 of SMARCA5 gene, and SMARCA5 gene has effect on regulating activities of helicase and ATPase, remodeling chromatin, facilitating the transcription of class II genes [9]. Previous published papers indicate that circ-SMARCA5 is downregulated and exerts anti-tumor effects in various cancers, such as hepatocellular carcinoma (HCC), non-small cell lung cancer, gastric cancer and cervical cancer [10-13]. For example, in HCC, circ-SMARCA5 is downregulated in HCC tissues, and its downregulation is associated with aggressive tumor features as well as unfavorable survival profiles in HCC patients [12, 14]. And in vivo experiments reveals that circ-SMARCA5 inhibits HCC cell proliferation and migration [14]. Considering that ICC and HCC share some similarities in molecular profiles, dominant risk factors and clinical manifestations, therefore, we speculated that circ-SMARCA5 might be involved in the ICC development and progression $[15,16]$. In the current study, we aimed to investigate the correlation of circSMARCA5 expression with clinical characteristics and survival profiles in ICC patients, and further conducted the cellular experiments to discover the role of circ-SMARCA5 in regulating cell proliferation and drug sensitivity of ICC.

\section{Methods}

\section{Patients}

This prospective study consecutively enrolled 92 primary ICC patients who underwent resection in our hospital between July 2014 and June 2016. The inclusion criteria were (i) newly diagnosed as primary ICC by imageology and histopathology; (ii) 18 years $\leq$ age $\leq 80$ years; (iii) clinical condition was suitable for surgery and about to receive resection; (iv) without other malignancies. The excluded criteria were: (i) HCC or mixed HCC-ICC; (ii) received neoadjuvant therapy before enrollment; (iii) with distant metastasis; (iv) history of human immunodeficiency virus infection; (v) pregnant or lactating women. This study was approved by Ethics Committee of our hospital and was conducted according to the principles expressed in the Declaration of Helsinki. All patients or their guardians provided the written informed consents.

\section{Data and sample collection}

The clinical characteristics of patients were recorded after enrollment, which included age, gender, smoke, drink, hepatitis B virus (HBV) infection, Eastern Cooperative Oncology Group (ECOG) performance score, pathological differentiation, T stage, N stage, TNM stage, carcinoembryonic antigen (CEA) level and carbohydrate antigen 199 (CA199) level. Tumor tissue and paired adjacent tissue resected from surgery were stored in liquid nitrogen, immediately. And the tumor tissue and adjacent tissue were divided into two parts, one was used for pathological diagnosis, another was used for circ-SMARCA5 detection in this study. 


\section{Reverse transcription quantitative polymerase chain reaction (RT-qPCR)}

The relative expression of circ-SMARCA5 in tumor tissue and adjacent tissue was detected by RT-qPCR. Total RNA was firstly extracted from tumor tissues and adjacent tissues using TRIzol Reagent (Invitrogen, USA), and linear RNA was removed using RNase R (Epicentre, USA). Following that, RNA was reversely transcribed to cDNA using iScript ${ }^{\text {tw }}$ cDNA Synthesis Kit (Bio-Rad, USA). Finally, qPCR was conducted using THUNDERBIRD ${ }^{\circledR}$ SYBR $^{\circledR}$ qPCR Mix (Toyobo, Japan). All the procedures were carried out according to the protocols of manufacturers. Then circ-SMARCA5 expression was calculated using $2^{-\triangle} \triangle \mathrm{Ct}$ with GAPDH as internal reference. The primer sequences used for RT-qPCR were listed as followed: Circ-SMARCA5 forward: ACAATGGATACAGAGTCAAG, reverse: CTTCATCAGTGATCTCACT; GAPDH forward: TGACCACAGTCCATGCCATCAC, reverse: GCCTGCTTCACCACCTTCTTGA.

\section{Grouping}

According to the relative expression of circ-SMARCA5 in tumor tissue, all patients were classified as circSMARCA5 high expression group (50\%-100\% quantile of circ-SMARCA5 relative expression in tumor tissue, $n=46)$ and circ-SMARCA5 low expression group (0\%-50\% quantile of circ-SMARCA5 relative expression in tumor tissue, $n=46$ ). In addition, the circ-SMARCA5 low expression group was further divided into circ-SMARCA5 low- expression group (25\%-50\% quantile of circ-SMARCA 5 relative expression in tumor tissue, $n=23)$, circ-SMARCA5 low- expression group (10\%-25\% quantile of circ-SMARCA5 relative expression in tumor tissue, $n=14)$ and circ-SMARCA5 low-- expression group $(0 \%-10 \%$ quantile of circSMARCA5 relative expression in tumor tissue, $n=9$ ).

\section{Treatment and follow-up}

After resection, all patients received gemcitabine/cisplatin combination adjuvant treatment based on the status of margins, according to NCCN Guidelines for Intrahepatic Cholangiocarcinoma [17]. Continuous follow-up was carried out for the patients until 2019/06/30. The median duration of follow-up was 26.5 months ranging from 2.0 to 60.0 months. Overall survival (OS) was defined as the duration from enrollment to death, and for the patients not known to have died in the last follow-up date, they were censored on the date of last known to be alive. Because most patients in the current study were non-local patients and the precise disease status of them was unbale to acquire timely, consequently, the diseasefree survival (DFS) was not evaluated.

\section{Cell culture}


Human ICC cell line TFK-1 was purchased from Deutsche Sammlung von Mikroorganismen und Zellkulturen (Braunschweig, German), while another human ICC cell line HuH-28 was purchased from Japanese Cancer Research Resources Bank - Cell Bank (Tokyo, Japan). TFK-1 cells were cultured in 90\% Roswell Park Memorial Institute (RPMI) 1640 Medium (Gibco, USA) and 10\% fetal bovine serum (FBS) (Gibco, USA) under $95 \%$ air $5 \% \mathrm{CO}_{2}$ at $37{ }^{\circ} \mathrm{C}$, while $\mathrm{HuH}-28$ cells were cultured in $80 \% \mathrm{RPMI} 1640$ Medium (Gibco, USA) and $20 \%$ FBS (Gibco, USA) under $95 \%$ air $5 \% \mathrm{CO}_{2}$ at $37^{\circ} \mathrm{C}$.

\section{Transfection}

The pCD 5-ciR vector (Geneseed Biotech Co., Ltd, China) was used to structure circ-SMARCA5 overexpression (OE) plasmid and control OE plasmid. After construction, the OE plasmids were transfected into HuH-28 cells and TFK-1 cells using Lipofectamine 2000 (Thermo, USA), then the cells transfected with circ-SMARCA5 OE plasmids were marked as OE-Circ group, correspondingly, the cells transfected with control OE plasmids were termed as OE-Control group. After transfection, cell proliferation in both groups were measured at 0h, 24h, 48h and 72h using Cell Counting Kit-8 (Sigma, USA), and the procedure was in accordance with manufacturer's manual.

\section{Drug sensitivity}

At $24 \mathrm{~h}$ after transfection, the OE-Circ cells and OE-Control cells were plated in 96 multi-well plates with a concentration of 5000 cells/well. Then the cells were treated with several concentrations of Cisplatin (Sigma, USA) and Gemcitabine (Sigma, USA) for 72h, respectively. After $72 \mathrm{~h}$ treatment, the cell viability of the OE-Circ cells and OE-Control cells at different drug concentrations was determined by Cell Counting Kit-8 (Sigma, USA), and the procedure was in accordance with manufacturer's manual. The cells treated with $0 \mu \mathrm{M}$ Cisplatin or $0 \mu \mathrm{M}$ Gemcitabine in each group were used as the reference in calculation of relative cell viability. Further, the drug concentration required to inhibit growth by $50 \%\left(\mathrm{IC}_{50}\right)$ was calculated for all treated cells with the use of Probit regression analysis.

\section{Statistical analysis}

Continuous variables were displayed as mean \pm standard deviation (SD) or median and interquartile range (IQR), and the categorical variables were expressed as count and percentage. Comparisons of unpaired variable between two groups were determined by Student's $t$ test or Chi-square test. Comparisons of paired variable between two groups were determined by Wilcoxon signed rank test. OS was illustrated using Kaplan-Meier curve, and the difference of OS between/among groups was determined by Log-rank test. Factors predicting OS were analyzed by univariate Cox's proportional hazard regression model and forward stepwise multivariate Cox's regression model. All statistical analyses were performed using SPSS 
22.0 software (IBM, USA), and figures were made using GraphPad Prism 7.00 software (GraphPad Software, USA). All tests were two-sided, $P$ value $<0.05$ was considered as significant.

\section{Results}

\section{Clinical characteristics of ICC patients}

In total ICC patients, the mean age was $59.2 \pm 7.4$ years, and there were $27(29.3 \%)$ females and 65 (70.7\%) males (Table 1). Regarding ECOG performance score, there were 57 (62.0\%) patients with 0, and 35 (38.0\%) patients with 1/2. As for pathological differentiation, there were 55 (59.8\%) patients with well/moderate pathological differentiation, and $37(40.2 \%)$ patients with poor pathological differentiation. The number of patients with I/II and III/IV TNM stage were 54 (58.7\%) and 38 (41.3\%) respectively. More detailed clinical characteristics were shown in Table 1.

\section{Comparison of Circ-SMAECA5 in ICC tumor tissues and pair adjacent tissues}

Circ-SMARCA5 relative expression was reduced in ICC tumor tissues $(0.311(0.179-0.602))$ compared with adjacent tissues $(0.987(0.709-1.297)(P<0.001)$ (Figure 1$)$.

\section{Correlation of circ-SMAECA5 expression with clinical features in ICC patients}

All ICC patients were divided into circ-SMARCA5 high expression group $(n=46)$ and circ-SMARCA5 low expression group ( $n=46)$ according to $50 \%$ quantile of circ-SMARCA5 relative expression in tumor tissue. Circ-SMARCA5 expression was negatively associated with ECOG performance score $(P<0.001)$, T stage $(P<0.036)$, N stage $(P<0.001)$, TNM stage $(P<0.001)$ and abnormal CA199 status $(P=0.019)$, however, there was no association of circ-SMARCA5 expression with age $(P=0.372)$, gender $(P=0.819)$, smoke $(P$ $=0.381)$, drink $(P=0.294)$, HBV infection $(P=0.393)$, pathological differentiation $(P=0.524)$ or CEA $(P$ $=0.524)$ in ICC patients (Table 1).

\section{Correlation of circ-SMARCA5 expression with OS in ICC patients}

OS was increased in patients with circ-SMARCA5 high expression compared with those with circSMARCA5 low expression $(P=0.007)$ (Figure 2A). Furthermore, circ-SMARCA5 low expression group was divided into circ-SMARCA5 low- expression group (25\%-50\% quantile of circ-SMARCA5 relative expression in tumor tissue), circ-SMARCA5 low- expression group (10\%-25\% quantile of circ-SMARCA5 relative expression in tumor tissue) and circ-SMARCA5 low-- expression group ( $0 \%-10 \%$ quantile of circSMARCA5 relative expression in tumor tissue). OS was the highest in patients with circ-SMARCA5 high 
expression, followed by patients with circ-SMARCA5 low- expression and patients with circ-SMARCA5 low- expression, and then patients with circ-SMARCA5 low- expression $(P<0.001)$ (Figure 2B).

\section{Factors affecting OS in ICC patients}

Univariate Cox's regression analysis exhibited that circ-SMARCA5 high expression ( $\mathrm{HR}=0.535, P=0.008)$ was associated with longer OS, while higher ECOG performance score (HR=2.060, $P=0.003)$, poor pathological differentiation ( $\mathrm{HR}=2.896, P<0.001)$, advanced TNM stage $(\mathrm{HR}=2.371, P<0.001)$ were correlated with reduced OS in ICC patients (Table 2). And forward stepwise multivariate Cox's regression analysis presented that circ-SMARCA5 high expression was an independent predictive factor for increased OS ( $\mathrm{HR}=0.518, P=0.006$ ), poor pathological differentiation was an independent predictive factor for decreased OS $(\mathrm{HR}=2.979, P<0.001)$ in ICC patients.

\section{Effect of circ-SMARCA5 upregulation on cell proliferation in ICC cells}

We conducted the in vitro experiments to explore the effect of circ-SMARCA5 upregulation on cell proliferation in two ICC cell lines. In HuH-28 ICC cells, cell proliferation was reduced in OE-Circ group compared with OE-Control group at $48 \mathrm{~h}(P<0.05)$ and $72 \mathrm{~h}(P<0.01)$ after transfection (Figure 3A). As for in TFK-1 cells, cell proliferation was also decreased in OE-Circ group compared with OE-Control group at 48h $(P<0.05)$ and $72 \mathrm{~h}(P<0.05)$ after transfection (Figure 3B).

\section{Effect of circ-SMARCA5 upregulation on chemotherapy sensitivity to cisplatin/gemcitabine in ICC cells}

Further experiments were performed to investigate the effect of circ-SMARCA5 upregulation on chemotherapy sensitivity to cisplatin/gemcitabine in two ICC cell lines. In HuH-28 cells, relative cell viability was decreased in OE-Circ group compared with OE-Control group in $4 \mu \mathrm{M}(P<0.05), 8 \mu \mathrm{M}(P$ $<0.05), 16 \mu \mathrm{M}(P<0.05)$ cisplatin-treated cells (Figure 4A); relative cell viability was also reduced in OE-Circ group compared with OE-Control group in $4 \mu \mathrm{M}(P<0.01), 8 \mu \mathrm{M}(P<0.01), 16 \mu \mathrm{M}(P<0.05)$ gemcitabinetreated cells (Figure 4B). Furthermore, $I_{50}$ value of cisplatin $(P<0.01)$ (Figure 4C) and gemcitabine $(P$ $<0.001$ ) (Figure 4D) were both decreased in OE-Circ group compared with OE-Control group. Similarly, in TFK-1 cells, relative cell viability was reduced in OE-Circ group compared with OE-Control group in $16 \mu \mathrm{M}$ $(P<0.05), 32 \mu \mathrm{M}(P<0.05)$ and $64 \mu \mathrm{M}(P<0.05)$ cisplatin-treated cells (Figure 4E); relative cell viability was also decreased in OE-Circ group compared with OE-Control group in $8 \mu \mathrm{M}(P<0.05), 16 \mu \mathrm{M}(P<0.05)$ gemcitabine-treated cells (Figure 4F). $\mathrm{IC}_{50}$ value of cisplatin $(P<0.01)$ (Figure $\left.4 \mathbf{G}\right)$ and gemcitabine $(P$ $<0.01$ ) (Figure 4H) were both decreased in OE-Circ compared with OE-Control. These suggested that circSMARCA5 upregulation promoted chemotherapy sensitivity to cisplatin/gemcitabine in ICC cells. 


\section{Discussion}

In the current study, we found that (1) circ-SMARCA5 was downregulated in ICC tumor tissues compared with adjacent tissues; (2) circ-SMARCA5 was negatively associated with ECOG performance score, TNM stage and abnormal CA199 status in ICC patients. (3) circ-SMARCA5 was an independent predictive factor for increased OS in ICC patients. (4) circ-SMARCA5 upregulation inhibited cell proliferation, and increased cell chemotherapy sensitivity to cisplatin/gemcitabine in ICC.

CircRNAs have been identified to be expressed stably in various cells, and they are reported to be involved in various physiological development, including serving as microRNA sponges, interacting with proteins, regulating transcription and splicing, participating in translation and etc. $[8,18,19]$. Until now, there are only preliminary investigations focusing on the role of circRNAs in cancers [19]. However, regarding circSMARCA5, as one of circRNAs, several publications reveal its participation in the tumorigenesis of various tumors, and circ-SMARCA5 high expression is reported to be correlated with favorable clinicopathological features and prognosis in patients with cancers $[10,11,13,20]$. For example, circSMARCS 5 is greatly downregulated in gastric cancer tissues compared with adjacent noncancerous tissues, and correlates with well differentiation, negative lymph node metastasis, no vascular invasion, and favorable prognosis in patients with gastric cancer [13]. In addition, circ-SMARCA5 is found to be downregulated in glioblastoma multiforme biopsies compared to normal brain tissues, and there exists a negative correlation between circ-SMARCA5 expression and histological grade in patients with glioma [20]. Furthermore, circ-SMARCA5 expression is decreased in HCC tissues compared with adjacent tissues, and its downregulation is correlated with advanced tumor features, furthermore, circ-SMARCA5 serves as an independent predictive factor for longer recurrence-free survival and OS in patients with HCC after hepatectomy [12]. However, the research on the role of circ-SMARCA5 in ICC has not conducted yet. Considering the similarities of cellular origin, genomic mutations, risk factors between ICC and HCC, we speculated that circ-SMARCA5 might also be aberrantly expressed in ICC tissues, and correlated with tumor features in ICC patients $[15,16]$. We found that circ-SMARCA5 was downregulated in ICC tumor tissues compared with adjacent tissues, and was negatively associated with ECOG performance score, TNM stage and abnormal CA199 status in ICC patients. The possible reasons might include that: CircSMARCA5 might inhibit cell proliferation, migration and invasion via acting as the miRNA sponge (such as: miR-17-3p and miR-181b-5p, which served as targets of circ-SMARCA5 in HCC) in ICC, which contributed to the tumor-inhibitory effect on ICC progression, therefore, circ-SMARCA5 is correlated with favorable clinicopathological features in ICC patients; however, this speculation needed to be further verified by cellular experiments in ICC [10]. In addition, as for the correlation of circ-SMARCA5 with survival profile in ICC patients, we observed that circ-SMARCA5 was an independent predictive factor for increased OS in ICC patients. This might be explained by that (1) circ-SMARCA5 was negatively associated with ECOG performance score, TNM stage and abnormal CA199 status in ICC patients, which were known as predictive factor for ICC prognosis. Therefore, circ-SMARCA5 might lead to desirable prognosis via interaction with these clinical-pathological tumor features. (2) Circ-SMARCA5 might be associated with SMARCA5-related functions (including: DNA repair and protection against the adverse effects of DNA damage) via activating DNA damage response, contributing to improved drug sensitivity, 
which was validated in our further functional experiments that circ-SMARCA5 increased cell chemotherapy sensitivity to cisplatin and gemcitabine in ICC. Thus, patients with circ-SMARCA5 high expression had increased survival in a long-term period compared with those with circ-SMARCA5 low expression [21].

Existing evidence demonstrates that circ-SMARCA5 are not simply by-products of gene splicing, but are of functionality and involved in regulating cell activities in various cancers $[10,11,14,20]$. For example, in cervical cancer, upregulation of circ-SMARCA5 inhibits cell proliferation, migration and invasion, and caused cell cycle arrest via being miR-620 sponge, suggesting the anti-tumor properties of circSMARCA5 [11]. In addition, circ-SMARCA5 promotes the expression of an anti-tumor gene, TIMP3, via functioning as the sponge of miR-181b and miR-17, resulting in inhibitory effect on HCC cell proliferation and migration [14]. However, the regulatory function of circ-SMARCA5 on cell activities in ICC has not been investigated yet. We found that upregulation of circ-SMARCA5 suppressed ICC cell proliferation, improved cell chemotherapy sensitivity to cisplatin as well as gemcitabine. The possible reasons might include that (1) Upregulation of circ-SMARCA5 might activate the expressions of antitumor genes (such as: TIMP3), which inhibited cell proliferation in ICC. (2) According to the previous study, cisplatin and gemcitabine are accepted as the standard chemotherapy regimen for ICC, and their abilities include involvement in DNA repair mechanisms, inducing cancer cell apoptosis, inhibitory effect in DNA damage $[2,22]$. Furthermore, circ-SMARCA5 might boost the DNA-repair ability and acquire resistance to DNA damaging agents via its located gene (SMARCA5), which reduced the formation of the drug resistance [21]. Thus circ-SMARCA5 upregulation might enhance the drug sensitivity (cisplatin and gemcitabine) in ICC cells. Combining the findings of cellular experiments and clinical research in our study, circ-SMARCA5 is suggested to be a candidate prognostic biomarker in ICC.

\section{Conclusions}

In conclusion, circ-SMARCA5 is downregulated in tumor tissues and correlates with favorable clinicopathological features and survival profile in ICC patients, and its upregulation not only inhibits proliferation, but also increases chemotherapy sensitivity to cisplatin and gemcitabine in ICC cells.

\section{Abbreviations}

ICC, Intrahepatic cholangiocarcinoma; circ-SMARCA5, CircRNA SWI/SNF related, matrix associated, actin dependent regulator of chromatin, subfamily a, member 5; HCC, hepatocellular carcinoma; HBV, hepatitis B virus; ECOG, Eastern Cooperative Oncology Group; CEA, carcinoembryonic antigen; CA199, carbohydrate antigen 199; RT-qPCR, Reverse transcription quantitative polymerase chain reaction; OS, Overall survival; DFS, disease-free survival; RPMI, Roswell Park Memorial Institute; FBS, fetal bovine serum; OE, overexpression; $I_{50}$, the drug concentration required to inhibit growth by $50 \%$; SD, standard deviation; IQR, interquartile range.

\section{Declarations}




\section{Ethics approval and consent to participate}

This study was approved by Ethics Committee of our hospital and was conducted according to the principles expressed in the Declaration of Helsinki. All patients or their guardians provided the written informed consents.

\section{Consent for publication}

Not applicable.

\section{Availability of data and material}

All data generated or analysed during this study are included in this published article.

\section{Competing interests}

The authors declare that they have no competing interests.

\section{Funding}

Not applicable.

\section{Authors' contributions}

LQ and FT designed and coordinated the study, made substantial contributions to the analysis, and drafted the manuscript. LQ performed statistical analysis. LQ and FT participated in data collection and interpretation. LQ helped to draft the manuscript. FT reviewed the manuscript and exerted a major impact on the interpretation of data and critical appraisal of the manuscript. All authors have read and approved the final manuscript.

\section{Acknowledgements}

Not applicable.

\section{References}


1.Razumilava N, Gores GJ. Cholangiocarcinoma. Lancet. 2014; 383:2168-79.

2.Chun YS, Javle M. Systemic and Adjuvant Therapies for Intrahepatic Cholangiocarcinoma. Cancer Control. 2017; 24:1073274817729241.

3.Ahn DH, Javle M, Ahn CW, Jain A, Mikhail S, Noonan AM et al. Next-generation sequencing survey of biliary tract cancer reveals the association between tumor somatic variants and chemotherapy resistance. Cancer. 2016; 122:3657-66.

4.Ribero D, Pinna AD, Guglielmi A, Ponti A, Nuzzo G, Giulini SM et al. Surgical Approach for Long-term Survival of Patients With Intrahepatic Cholangiocarcinoma: A Multi-institutional Analysis of 434 Patients. Arch Surg. 2012; 147:1107-13.

5.Hezel AF, Zhu AX. Systemic therapy for biliary tract cancers. Oncologist. 2008; 13:415-23.

6.Hezel AF, Deshpande V, Zhu AX. Genetics of biliary tract cancers and emerging targeted therapies. J Clin Oncol. 2010; 28:3531-40.

7.Weber SM, Ribero D, O’Reilly EM, Kokudo N, Miyazaki M, Pawlik TM. Intrahepatic cholangiocarcinoma: expert consensus statement. HPB (Oxford). 2015; 17:669-80.

8.Kristensen LS, Hansen TB, Veno MT, Kjems J. Circular RNAs in cancer: opportunities and challenges in the field. Oncogene. 2018; 37:555-65.

9.LeRoy G, Orphanides G, Lane WS, Reinberg D. Requirement of RSF and FACT for transcription of chromatin templates in vitro. Science. 1998; 282:1900-4.

10.Wang Y, Li H, Lu H, Qin Y. Circular RNA SMARCA5 inhibits the proliferation, migration, and invasion of non-small cell lung cancer by miR-19b-3p/HOXA9 axis. Onco Targets Ther. 2019; 12:7055-65.

11.Tian JDC, Liang L. Involvement of circular RNA SMARCA5/microRNA-620 axis in the regulation of cervical cancer cell proliferation, invasion and migration. Eur Rev Med Pharmacol Sci. 2018; 22:8589-98.

12.Yu J, Xu QG, Wang ZG, Yang Y, Zhang L, Ma JZ et al. Circular RNA cSMARCA5 inhibits growth and metastasis in hepatocellular carcinoma. J Hepatol. 2018; 68:1214-27.

13.Cai J, Chen Z, Zuo X. circSMARCA5 Functions as a Diagnostic and Prognostic Biomarker for Gastric Cancer. Dis Markers. 2019; 2019:2473652.

14.Li Z, Zhou Y, Yang G, He S, Qiu X, Zhang L et al. Using circular RNA SMARCA5 as a potential novel biomarker for hepatocellular carcinoma. Clin Chim Acta. 2019; 492:37-44.

15.Lendvai G, Szekerczes T, Illyes I, Dora R, Kontsek E, Gogl A et al. Cholangiocarcinoma: Classification, Histopathology and Molecular Carcinogenesis. Pathol Oncol Res. 2018. 
16.Wang $\mathrm{M}$, Gao Y, Feng H, Warner E, An M, Jia J et al. A nomogram incorporating six easily obtained parameters to discriminate intrahepatic cholangiocarcinoma and hepatocellular carcinoma. Cancer Med. $2018 ; 7: 646-54$.

17.NCCN Clinical Practice Guidelines in Oncology: Hepatobiliary Cancers (Version 2.2014).

18.Xu Y, Yao Y, Liu Y, Wang Z, Hu Z, Su Z et al. Elevation of circular RNA circ_0005230 facilitates cell growth and metastasis via sponging miR-1238 and miR-1299 in cholangiocarcinoma. Aging (Albany NY). 2019; 11:1907-17.

19.Merdrignac A, Angenard G, Allain C, Petitjean K, Bergeat D, Bellaud P et al. A novel transforming growth factor beta-induced long noncoding RNA promotes an inflammatory microenvironment in human intrahepatic cholangiocarcinoma. Hepatol Commun. 2018; 2:254-69.

20.Barbagallo D, Caponnetto A, Cirnigliaro M, Brex D, Barbagallo C, D'Angeli F et al. CircSMARCA5 Inhibits Migration of Glioblastoma Multiforme Cells by Regulating a Molecular Axis Involving Splicing Factors SRSF1/SRSF3/PTB. Int J Mol Sci. 2018; 19.

21.Aydin OZ, Vermeulen W, Lans H. ISWI chromatin remodeling complexes in the DNA damage response. Cell Cycle. 2014; 13:3016-25.

22.Dasari S, Tchounwou PB. Cisplatin in cancer therapy: molecular mechanisms of action. Eur J Pharmacol. 2014; 740:364-78.

\section{Tables}

\section{Table 1. Clinical characteristics}




\begin{tabular}{|c|c|c|c|c|}
\hline \multirow[t]{2}{*}{ Items } & \multirow{2}{*}{$\begin{array}{l}\text { Total patients } \\
\qquad(\mathrm{N}=92)\end{array}$} & \multicolumn{2}{|c|}{ Circ-SMARCA5 } & \multirow{2}{*}{$\begin{array}{c}P \\
\text { value }\end{array}$} \\
\hline & & $\begin{array}{c}\text { Low } \\
(\mathrm{n}=46)\end{array}$ & $\begin{array}{l}\text { High } \\
(\mathrm{n}=46)\end{array}$ & \\
\hline Age (years), mean \pm SD & $59.2 \pm 7.4$ & $58.5 \pm 8.0$ & $59.9 \pm 6.8$ & 0.372 \\
\hline Gender, No. (\%) & & & & 0.819 \\
\hline Female & $27(29.3)$ & $13(28.3)$ & $14(30.4)$ & \\
\hline Male & $65(70.7)$ & $33(71.7)$ & $32(69.6)$ & \\
\hline Smoke, No. (\%) & $32(34.8)$ & $18(39.1)$ & $14(30.4)$ & 0.381 \\
\hline Drink, No. (\%) & $41(44.6)$ & $18(39.1)$ & $23(50.0)$ & 0.294 \\
\hline HBV infection, No. (\%) & $36(39.1)$ & $16(34.8)$ & $20(43.5)$ & 0.393 \\
\hline $\begin{array}{l}\text { ECOG performance score, No. } \\
(\%)\end{array}$ & & & & $<0.001$ \\
\hline 0 & $57(62.0)$ & $20(43.5)$ & $37(80.4)$ & \\
\hline $1 / 2$ & $35(38.0)$ & $26(56.5)$ & 9 (19.6) & \\
\hline $\begin{array}{l}\text { Pathological differentiation, No. } \\
\text { (\%) }\end{array}$ & & & & 0.524 \\
\hline Well and moderate & $55(59.8)$ & $26(56.5)$ & $29(63.0)$ & \\
\hline Poor & $37(40.2)$ & $20(43.5)$ & $17(37.0)$ & \\
\hline T stage, No. (\%) & & & & 0.036 \\
\hline $\mathrm{T} 1 / \mathrm{T} 2$ & $74(80.4)$ & $33(71.7)$ & $41(89.1)$ & \\
\hline T3/T4 & $18(19.6)$ & $13(28.3)$ & $5(10.9)$ & \\
\hline N stage, No. (\%) & & & & $<0.001$ \\
\hline No & $56(60.9)$ & $17(37.0)$ & $39(84.8)$ & \\
\hline N1 & $36(39.1)$ & $29(63.0)$ & $7(15.2)$ & \\
\hline TNM stage, No. (\%) & & & & $<0.001$ \\
\hline $\mathrm{I} / \mathrm{II}$ & $54(58.7)$ & $17(37.0)$ & $37(80.4)$ & \\
\hline III/IV & $38(41.3)$ & $29(63.0)$ & $9(19.6)$ & \\
\hline CEA abnormal*, No. (\%) & $37(40.2)$ & $20(43.5)$ & $17(37.0)$ & 0.524 \\
\hline CA199 abnormal ${ }^{\#}$, No. (\%) & $55(59.8)$ & $33(71.7)$ & $22(47.8)$ & 0.019 \\
\hline
\end{tabular}

Comparison was determined by Student's t test or Chi-square test. ICC, intrahepatic cholangiocarcinoma; SD, standard deviation; HPV, hepatitis B virus; ECOG, Eastern Cooperative Oncology Group; CEA, carcinoembryonic antigen; CA199, carbohydrate antigen 199. *abnormal, CEA $>5 \mathrm{ng} / \mathrm{mL}$; normal, CEA $\leq 5 \mathrm{ng} / \mathrm{mL}$; ${ }^{*}$ abnormal, CA199>37 $\mathrm{U} / \mathrm{mL}$; normal, CA199 $\leq 37 \mathrm{U} / \mathrm{mL}$.

Table 2. Analysis of factors predicting OS 


$$
P \text { value }
$$

HR

95\%CI

Lower

Higher

Univariate Cox's regression

Circ-SMARCA5

Low expression

Reference

High expression

0.008

0.535

0.336

0.850

Age

$\leq 60$ years

Reference

$>60$ years

0.713

1.089

0.691

1.717

Gender

Female

Reference

Male

0.689

1.113

0.659

1.878

Smoke

No

Reference

Yes

0.206

1.348

0.848

2.143

Drink

No

Reference

Yes

0.859

1.042

0.660

1.646

HBV infection

No

Reference

Yes

0.902

1.030

0.643

1.651

ECOG performance score

0

$1 / 2$

Pathological differentiation

Well/moderate

Poor

TNM stage

I/II
Reference

0.003

Reference

$$
<0.001
$$

2.896

1.787

4.692
Reference 
III/IV

$$
<0.001
$$

2.371

1.484

3.787

CEA *

Normal

Reference

Abnormal

0.143

1.414

0.889

2.247

CA199 \#

Normal

Reference

Abnormal

0.130

1.438

0.899

2.303

Forward stepwise multivariate Cox's regression Circ-SMARCA5

Low expression

High expression
Reference

0.006

Reference

$$
<0.001
$$

Pathological differentiation (poor)

Well/moderate

2.979

1.828

4.855

Factors predicting OS were analyzed by univariate Cox's proportional hazard regression model and forward stepwise multivariate Cox's regression model. OS, overall survival; HR, hazard ratio; CI, confidence interval; HPV, hepatitis B virus; ECOG, Eastern Cooperative Oncology Group; CEA, carcinoembryonic antigen; CA199, carbohydrate antigen 199.

CEA>5 ng/mL; normal, CEA $\leq 5$ ng/mL; ${ }^{*}$ abnormal, CA199>37 U/mL; normal, CA199 37 $\mathrm{U} / \mathrm{mL}$.

\section{Figures}




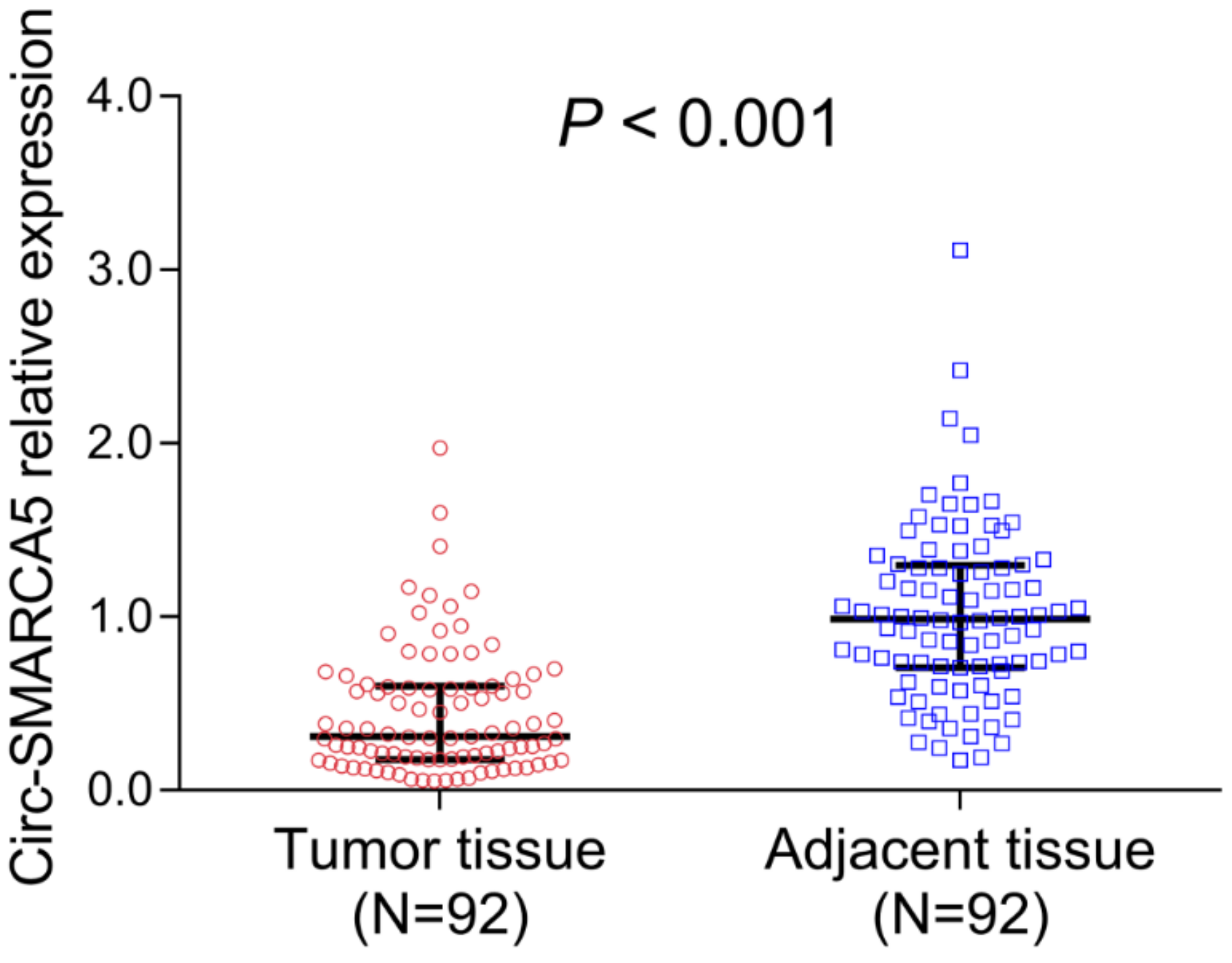

Figure 1

Circ-SMARCA5 expression was reduced in ICC tumor tissue compared with adjacent tissue. ICC, intrahepatic cholangiocarcinoma; Circ-SMARCA5, circular RNA SWI/SNF related, matrix associated, actin dependent regulator of chromatin, subfamily a, member 5 .

A

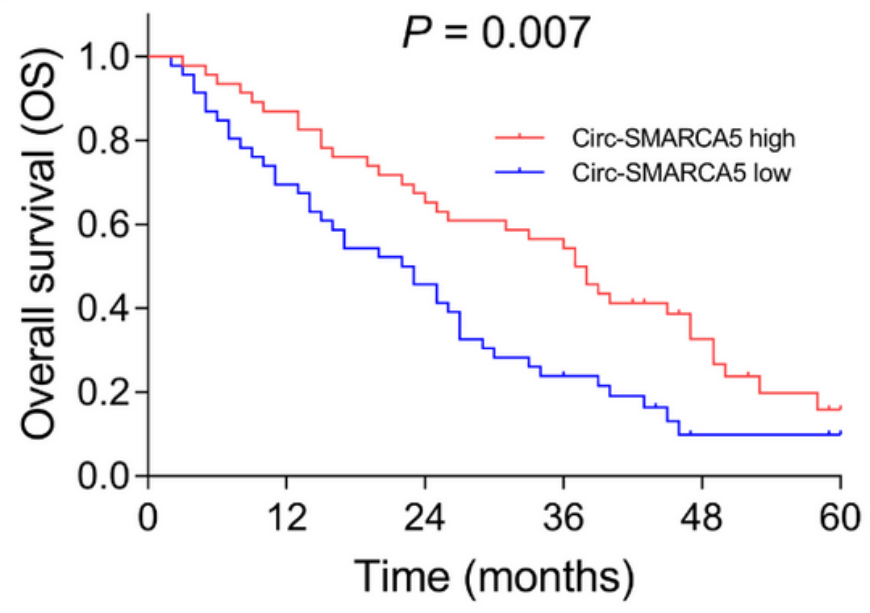

B

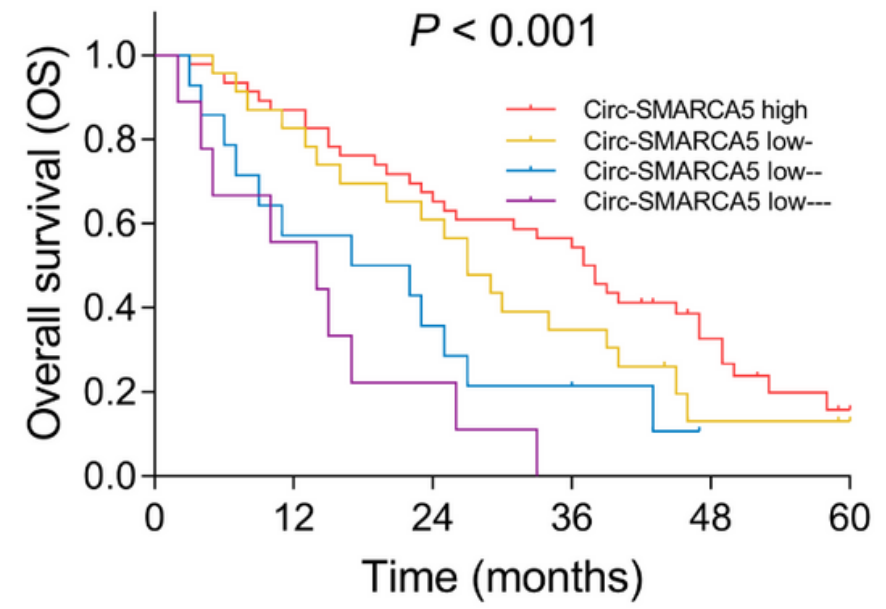


Figure 2

Circ-SMARCA5 positively associated with OS in ICC patients. Comparison of OS between ICC patients with circ-SMARCA5 high expression and those with circ-SMARCA5 low expression (A). Comparison of OS among ICC patients with circ-SMARCA5 high expression, those with low- expression, those with lowexpression and those with low--expression (B). OS, overall survival; ICC, intrahepatic cholangiocarcinoma; circ-SMARCA5, circular RNA SWI/SNF related, matrix associated, actin dependent regulator of chromatin, subfamily a, member 5 ; circ-SMARCA5 low- expression, $25 \%-50 \%$ quantile of circ-SMARCA5 relative expression in tumor tissue; circ-SMARCA5 low- expression, $10 \%-25 \%$ quantile of circ-SMARCA5 relative expression in tumor tissue; circ-SMARCA5 low- expression, 0\%-10\% quantile of circ-SMARCA5 relative expression in tumor tissue.

A

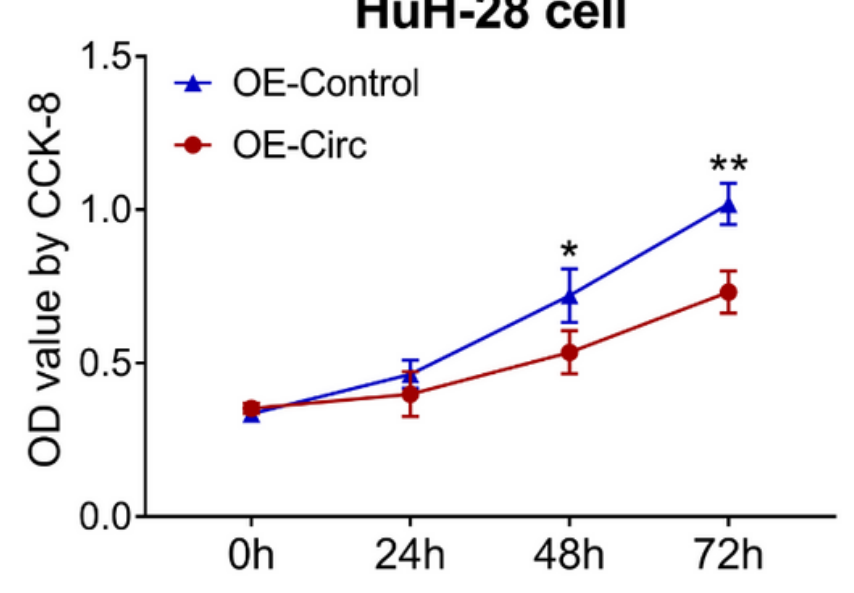

B

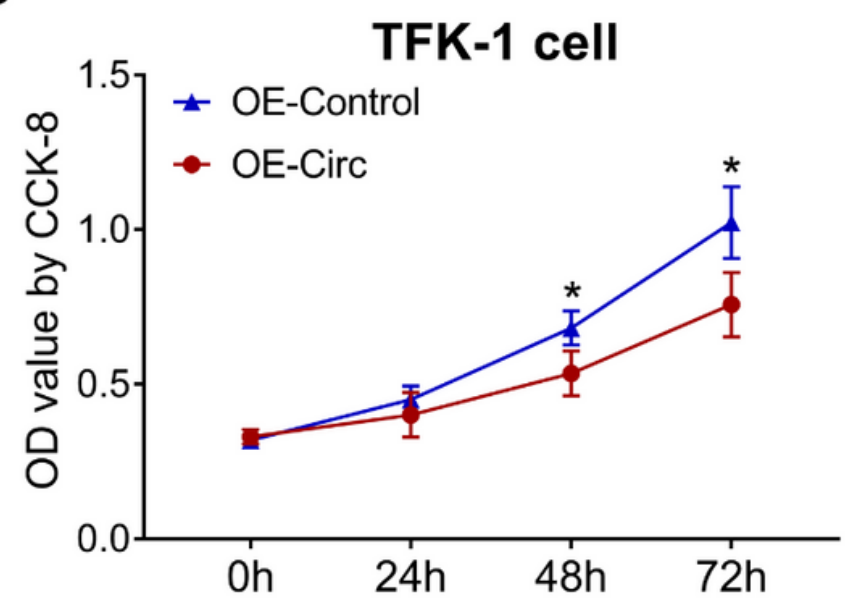

\section{Figure 3}

Circ-SMARCA5 inhibited proliferation in ICC cells after transfection. Comparison of OD value by CCK-8 between OE-Control group and OE-Circ in HuH-28 cells (A) and TFK-1 cells (B). ICC, intrahepatic cholangiocarcinoma; circ-SMARCA5, circular RNA SWI/SNF related, matrix associated, actin dependent regulator of chromatin, subfamily a, member 5 ; OE-Circ group, the cells transfected with circ-SMARCA5 overexpression plasmids; OE-Control group, the cells transfected with control overexpression plasmids; OD, optical density, CCK-8, Cell Counting Kit-8. 

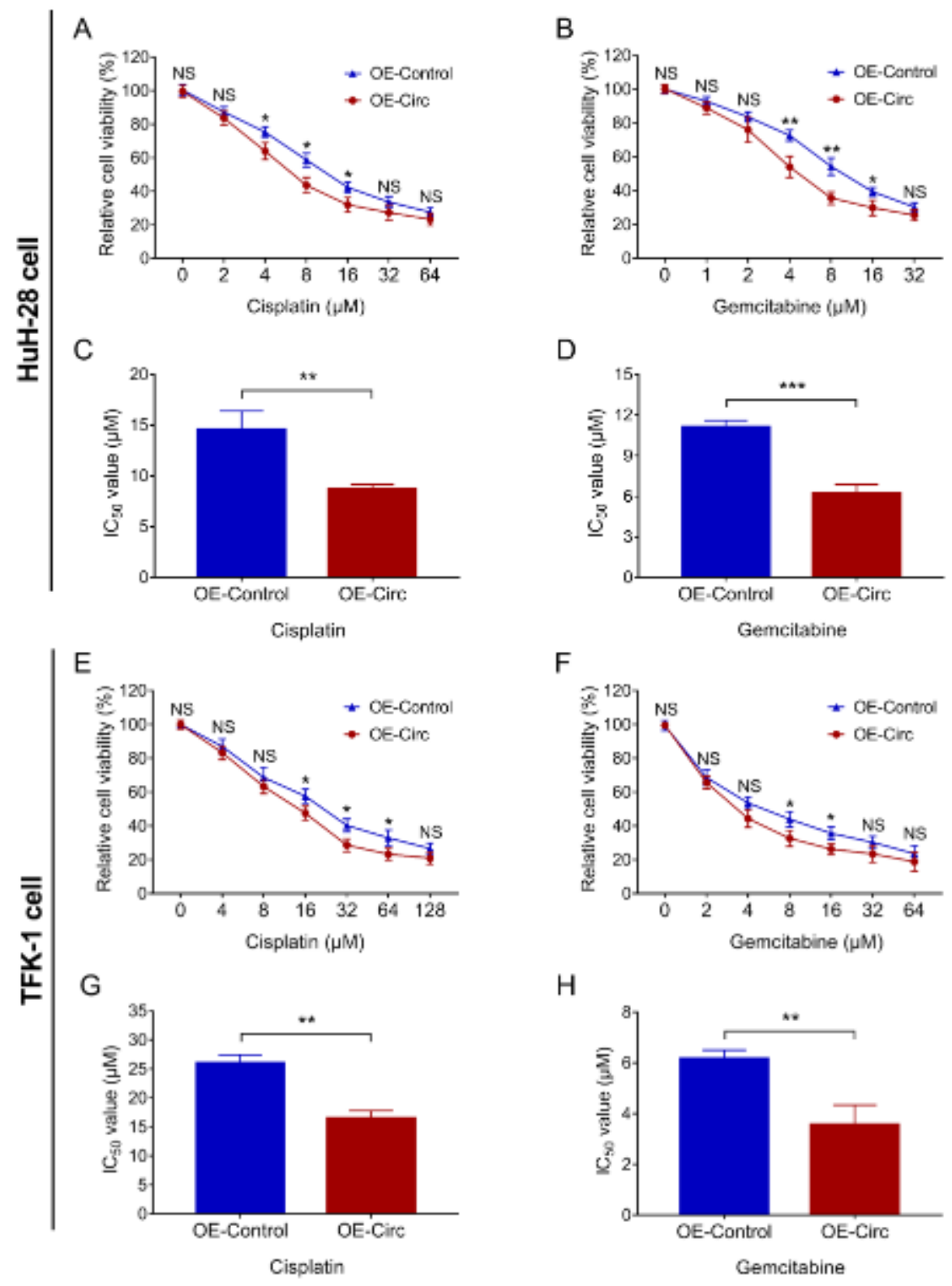

Figure 4

Circ-SMARCA5 increased chemotherapy sensitivity to cisplatin/gemcitabine in ICC cells after transfection. Comparison of relative cell viability between OE-Control group and OE-Circ group treated by $0 \mu \mathrm{M}, 2 \mu \mathrm{M}, 4 \mu \mathrm{M}, 8 \mu \mathrm{M}, 16 \mu \mathrm{M}, 32 \mu \mathrm{M}, 64 \mu \mathrm{M}$ cisplatin (A) and gemcitabine (B) in HuH-28 cells. Comparison of IC50 value of cisplatin (C) and gemcitabine (D) between OE-Control group and OE-Circ group in HuH-28 cells. Comparison of relative cell viability between OE-Control group and OE-Circ group treated by $0 \mu \mathrm{M}$, $4 \mu \mathrm{M}, 8 \mu \mathrm{M}, 16 \mu \mathrm{M}, 32 \mu \mathrm{M}, 64 \mu \mathrm{M}, 128 \mu \mathrm{M}$ cisplatin (E) and gemcitabine (F) in TFK-1 cells. Comparison of IC50 value of cisplatin $(\mathrm{G})$ and gemcitabine $(\mathrm{H})$ between OE-Control group and OE-Circ group in TFK-1 cells. ICC, intrahepatic cholangiocarcinoma; circ-SMARCA5, circular RNA SWI/SNF related, matrix associated, actin dependent regulator of chromatin, subfamily a, member 5; OE-Circ group, the cells transfected with circ-SMARCA5 overexpression plasmids; OE-Control group, the cells transfected with control overexpression plasmids; IC50, drug concentration required to inhibit growth by $50 \%$. 